\title{
A Stochastic Analysis of Pumping Tests in Laterally Nonuniform Media
}

\author{
JAMES J. BUTLER, JR. ${ }^{1}$ \\ Kansas Geological Survey, University of Kansas, Lawrence
}

\begin{abstract}
Conventional pumping test analysis methodology assumes that aquifer transmissivity is invariant in space. The ramifications of this assumption are examined for hypothetical units whose variations in transmissivity are considered to be reasonable representations of variations possible in natural systems. The dependence of pumping test transmissivity on spatial (angular and radial) and temporal location of observations and the method of drawdown analysis is assessed. The dependence on the angular position of an observation well appears of little significance. Dependence on radial position, however, can be strong. The dependence on the interval of time used in the pumping test analysis is only important in highly variable systems. Methods of drawdown analysis that yield identical estimates in uniform units yield differing estimates in nonuniform ones as a result of a difference in data-fitting procedures. A reasonable estimate of transmissivity at a regional scale can be obtained from a Cooper-Jacob analysis applied at a large duration of pumpage. In general, conventional approaches for pumping test analysis should be viable in the nonuniform aquifers of the type considered here.
\end{abstract}

\section{INTRODUCTION}

A pumping test is the primary means of assessing the transmissive and storage properties of subsurface material on a scale of significance for issues involving water supply or the gross movement of a contaminant plume. The parameters characterizing the transmissive and storage nature of a unit are estimated from pumping-induced drawdown using analytical solutions to the partial differential equation goveming the flow of groundwater to a central pumping well. These analytical solutions assume that the aquifer is uniform or can be subdivided into, at most, two or three uniform regions [Streltsova, 1988]. Although most hydrogeologists recognize that natural systems are characterized by a considerable degree of spatial variability, only limited work has been done to evaluate the ramifications of that variability for pumping test analyses [e.g., Warren and Price, 1961; Vandenberg, 1977]. This article describes an attempt to address these ramifications in more depth than previous work.

The major purpose of the work reported here is to examine theoretically the applicability of conventional pumping test analysis methodology, developed for uniform units, to units whose properties vary in space. Specifically, this examinaton focuses on the impact of lateral variations in the transmissive properties of a unit on the transmissivity estimates obtained from conventional pumping test analysis methodology. The impact of these lateral variations is quantified using the dependence of the estimated transmissivity on the spatial and temporal location of the drawdown data employed in the analysis (in this work spatial location refers to position of the observation well, and temporal location refers to the interval of time represented by the drawdown data). Only behavior in perfectly confined aquifers is considered here. This simplification allows the effect of lateral vanations in transmissivity to be more readily evaluated.

The emphasis of this study is on conditions in units that are representative of those that might be found in natural

\footnotetext{
Formerly at the Department of Applied Earth Sciences, Stanford University, Stanford, California.

Ceprright 1991 by the American Geophysical Union.

Raper number 91WR01371.

1397/91/91WR-01371\$05.00
}

systems. Thus an effort is made to employ mathematical representations of units whose spatial variations in transmissivity can be considered to be reasonably similar to those of aquifers in the field. Since detailed deterministic descriptions of variations in transmissivity over a field area are not available, a stochastic process representation is employed to incorporate the limited existing field data into this study. An important advantage of a stochastic representation is that it allows the uncertainty concerning the actual spatial variations in natural systems to be incorporated into the analysis. The results of this work are therefore phrased in terms of behavior in the class of units that can be represented by a specific stochastic process.

Since conventional pumping test analysis methodology is often used to provide estimates of the regional properties of a flow system, this work also examines the viability of the transmissivity calculated from a pumping test analysis for use in a regional flow model. The aim of this aspect of the work is to define appropriate procedures for estimation of the regional transmissivity from drawdown data collected during a pumping test.

\section{METHODOLOGY}

The basic approach of this research is to numerically simulate a pumping test in a realization drawn from a stochastic process characterizing the variations in transmissivity over the modeled area. A pumping test transmissivity is then estimated from the simulated drawdown data using conventional analytical approaches. The dependence of the estimated transmissivity on the spatial and temporal location of observations, and the method of drawdown analysis (the various forms of transmissivity dependence are henceforth denoted as dependence relationships), is then calculated for that realization. Monte Carlo simulation is employed to repeat this procedure over a sample of realizations drawn from the stochastic process. The average and variability of the dependence relationships over the realizations drawn from the stochastic process are then used to assess the viability of conventional pumping test analysis methodology in the class of units represented by that process. Four stochastic processes are examined in the work discussed here. Given the large computational demands of Monte 
Carlo simulation, the emphasis of this study will be on obtaining a general understanding of these dependence relationships, rather than the definition of highly precise expressions describing their form.

A finite element model, the FE3DGW model of Gupta et al. [1984], is employed for the simulations of this work. Equation (1) is the form of the flow equation used here:

$\left(\frac{\partial T}{\partial r}\right)\left(\frac{\partial \bar{s}}{\partial r}\right)+T \frac{\partial^{2} \bar{s}}{\partial r^{2}}+\frac{T}{r} \frac{\partial \bar{s}}{\partial r}+\frac{1}{r^{2}} \frac{\partial}{\partial \theta}\left(T \frac{\partial \bar{s}}{\partial \theta}\right)=S \frac{\partial \bar{s}}{\partial t}$

where

$T$ transmissivity, equal to $\bar{K} B, L^{2} / T$;

$S$ storage coefficient, equal to $\bar{S}_{0} B$, dimensionless;

$\bar{s}$ integrated vertical average of drawdown, $L$;

$B$ aquifer thickness, $L$;

$\bar{K}, \bar{S}_{0}$ integrated vertical average of hydraulic conductivity and specific storage, respectively;

$r$ radial direction, $L$;

$\theta$ angular direction, rad.

The initial and boundary conditions used in the simulations are

$$
\begin{gathered}
\bar{s}(r, \theta, 0)=0 \quad r_{w}<r<r_{b} \quad 0 \leq \theta \leq 2 \pi \\
\bar{s}\left(r_{b}, \theta, t\right)=0 \quad 0 \leq \theta \leq 2 \pi \quad t>0 \\
r_{w} T \int_{0}^{2 \pi}\left(\frac{\partial \bar{s}}{\partial r}\right)_{r_{w}} d \theta=-Q \quad t>0
\end{gathered}
$$

where $Q$ is the pumpage from the central well, $L^{3} / T ; r_{w}$ is the radius of the pumping well, $L$; and $r_{b}$ is the radius of the outer aquifer boundary, $L$.

For the simulations of this work a pumping well with a radius of $0.07 \mathrm{~m}$, an outer aquifer boundary that is $1930 \mathrm{~m}$ from the pumping well, and a pumpage rate of $6250 \mathrm{~m}^{3} / \mathrm{d}$ are employed. The finite element grid, which consists of quadrilateral elements, utilizes an equal log spacing in the radial direction, with approximately four elements per log cycle. Sixteen elements are employed in the angular direction. A pumping period of 2.7 days is used in the majority of the simulations, a duration considered adequate to represent conditions faced in many pumping tests. One set of simulations is carried out for a significantly longer duration (43.25 days) in order to evaluate behavior at very large times. In neither case does the constant head boundary at $1930 \mathrm{~m}$ significantly influence the observation well drawdown.

Drawdown data are simulated for the observation well network depicted in Figure 1. The drawdown record at each well is then used to obtain a separate estimate for the transmissivity of the aquifer. Storativity is assumed a known constant throughout this work. The approaches used for the transmissivity estimation are based on the analytical solution by Theis [1935] to the following equation and auxiliary conditions describing radial flow to a pumping well in a uniform aquifer:

$$
T \frac{\partial^{2} \bar{s}}{\partial r^{2}}+\frac{T}{r} \frac{\partial \bar{s}}{\partial r}=S \frac{\partial \bar{s}}{\partial t}
$$

Initial conditions

$$
\bar{s}(r, 0)=0 \quad r_{w^{\prime}}<r<\infty
$$

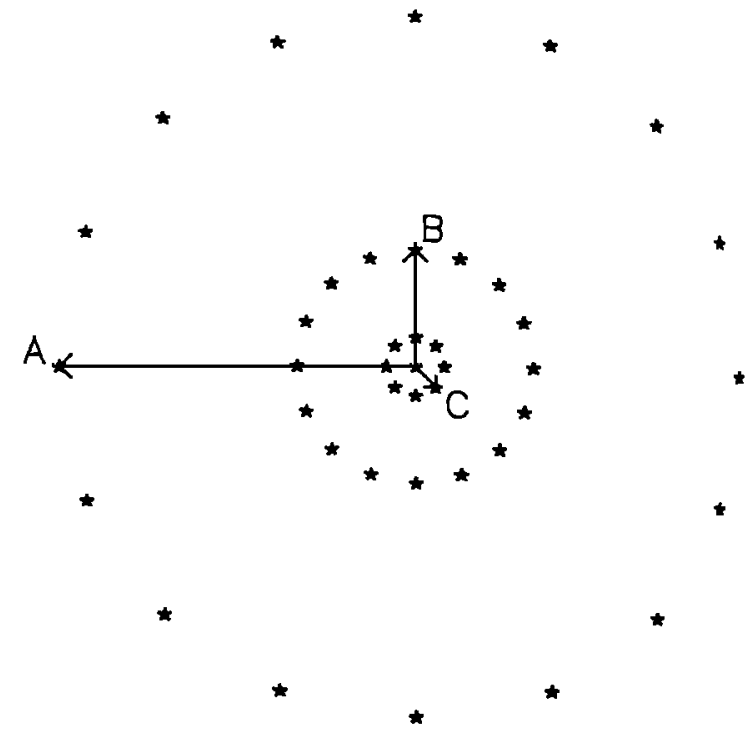

Fig. 1. Observation well network used in this study $\left(r_{A}=49.6\right.$ $\mathrm{m}, r_{B}=12.6 \mathrm{~m}, r_{C}=1.3 \mathrm{~m}$, not to scale); the flow model has and elements over $r_{C}$, five elements between $r_{C}$ and $r_{B}$, and three elements between $r_{B}$ and $r_{A}$. Sixteen wells are spaced at equal intervals along the circumference of each circle of observation wells, although only eight are shown for $r_{C}$.

Boundary conditions

$$
\begin{gathered}
\lim _{r_{w} \rightarrow 0} 2 \pi r_{w} T \frac{\partial \bar{s}}{\partial r}=-Q \quad t>0 \\
\bar{s}(\infty, t)=0 \quad t>0
\end{gathered}
$$

An unweighted nonlinear least squares minimization approach employing Theis' solution to (3) and (4) and automated analogue of the Cooper-Jacob semilog procedrat [Cooper and Jacob, 1946] are the two estimation approaches used here. The nonlinear least squares (NLS) routine volves fitting a theoretical drawdown versus time curve frot the solution of (3) and (4) to the drawdown data. The fittim is done using untransformed drawdown and time. The $C_{00}$ per-Jacob (C-J) procedure involves fitting a straight line to the moderate- and large-time drawdown data on a semilo plot. In both procedures, simulated drawdown are analyze over intervals of approximately one, two, and three loganth mic cycles in time. For the one set of simulations represext ing a pumping test of 43 days in duration, an additional analysis over an interval of four log cycles in time is performed. In all cases the drawdown analyses begin at an initial time of 0.0023 days. Simulated drawdown at earier times is ignored in order to avoid using data from the penod when well bore storage effects are present [Papadopulos and Cooper, 1967]. Error introduced by the spatial and tempord discretization schemes employed in the finite element model propagate into the transmissivities estimated from the sints lated drawdown, requiring the correction procedure described in Appendix $\mathrm{C}$ be applied before the transmissivitics can be used further.

Several functions are employed in order to quantify dependence of pumping test transmissivity on the angur. radial, and temporal location of observations and the methor of drawdown analysis. Angular dependence in transmissiva 
values is assessed using a coefficient of variation and the romalized absolute value of the transmissivity difference. This coefficient of variation consists of the standard deviaron of transmissivity values calculated at individual wells bout the circumference of a circle of observation wells (see Figure 1) over the mean of these values. The normalized frerence consists of the absolute difference between transwissivity values calculated at two wells spaced at a given mular separation normalized by the value at the first well. Both wells are at the same radial distance from the pumping ell. In this paper the coefficient of variation is primarily caployed for describing the angular dependence, since it more succinctly summarizes the studied relationships. Note the the observation well network displayed in Figure 1 results in drawdown being measured at equal angular intervals at different radial distances from the pumping well. yeasurement at equal angular intervals produces sampling schemes that are not strictly comparable from one circle of observation wells to the next, because the sampling interval relative to the correlation structure of the stochastic process icreases with radial distance from the pumping well. Given the goals of this work, however, the differences between sampling schemes are considered insignificant.

Radial and temporal dependence are characterized by the mormalized absolute value of the transmissivity difference. In each case a transmissivity value calculated from drawdown at the pumping well is considered the base value for the realization. The absolute value of the difference between this base value and a transmissivity computed at a different redial or temporal location is normalized by the base value. The normalized absolute value of the transmissivity difference is also used to characterize parameter dependence on the method of drawdown analysis, with the NLS transmissivity being used as the normalizing value for the difference between the NLS and C-J parameters.

The mean and standard deviation of these functions over a stochastic process are the quantities of interest in this work. Ia onder to estimate these statistics, the procedure of pumptest simulation and drawdown analysis is repeated for a saple of realizations drawn from a given stochastic process. The TUBA program [Mantoglou and Wilson, 1981], wich implements a two-dimensional form of the turning bands method of Matheron [1973], is used for the generation of realizations of spatially correlated transmissivity values. The TUBA program generates rectangular elements in a Cartesian grid system. As is described in Appendix A, the Cartesian coordinate output from the TUBA program is transformed into the polar coordinate grid system used in the pumping test simulations. A stratified sampling scheme described in Appendix $\mathbf{A}$ is employed to reduce the number of realizations required for the repetitive Monte Carlo simwation. A stratified sample of twenty realizations is used in all but one of the cases examined here. This sampling procedure is considered appropriate, given the earlier-stated craphasis of this work on general understanding rather than detailed description.

The stochastic processes employed in this work are based the Quadra Sands data of Smith [1978]. As is described in Appendix B, the core-scale data of Smith [1978] are regularirod to the scale of a block $10 \mathrm{~m}$ by $10 \mathrm{~m}$ in lateral extent in ander to reduce the computational demands of the realizageneration procedure. The stochastic process defined by the regularized Quadra Sands data is then modified by
TABLE 1. Stochastic Processes Employed in This Work

\begin{tabular}{cl}
\hline Case Number & \multicolumn{1}{c}{ Process } \\
\hline 1 & $E\{Y\}=-3.32$ \\
& $\gamma(r)=0.04537\left(1-e^{\left.-(r / 380 .)^{2}\right)}\right.$ \\
& $E\{Y\}=-3.32$ \\
& $\gamma(r)=0.04537\left(1-e^{\left.-(r / 95 .)^{2}\right)}\right.$ \\
3 & $E\{Y\}=-3.32$ \\
4 & $\gamma(r)=0.1589\left(1-e^{-(r / 95 .)^{2}}\right)$ \\
& $E\{r\}=-3.32$ \\
& $X(r)=0.3452\left(1-e^{\left.-(r / 95 .)^{2}\right)}\right.$ \\
\hline
\end{tabular}

$E\{\}$ is the expected value operator; $Y=\ln \bar{K}, \bar{K}$ (hydraulic conductivity averaged over a 10 by 10 by $30 \mathrm{~m}$ ( $x y z)$ block) is measured in centimeters per second: and $\gamma(r)$ is the semivariogram value for separation distance $r$ (see (B1) and (B4) of Appendix B for further details). Since all the stochastic processes are Gaussian, isotropic, and second-order stationary, the expected value and semivariogram expressions given for each case are sufficient to fully describe that process. A double-exponential semivariogram model is employed for all cases in order to allow the greater continuity at the origin produced by regularization to be included in the analysis.

decreasing the distance over which values are correlated and increasing the variance in order to allow behavior under conditions of greater variability to be assessed. These modifications are based on geologic considerations and a literature review of existing laboratory and field data. Table 1 lists the Quadra Sands-based stochastic process (case 1) and the modifications used in this work. The stochastic processes of Table 1 are written in terms of the hydraulic conductivity averaged over the block. Given the aquifer thickness of $30 \mathrm{~m}$ used here, this translates into an expected value for transmissivity between 959 and $1114 \mathrm{~m}^{2} / \mathrm{d}$ for the four cases examined in this work. The span of conditions represented by these four cases allows pumping test behavior in laterally nonuniform units to be assessed over conditions that might be met in the field. Further details concerning these cases and the motivation for their selection can be found in Appendix B. Note that a constant storativity of 0.0795 is used throughout this work.

\section{RESULTS}

\section{Dependence on Angular Location}

The dependence of pumping test transmissivity on the angular location of observation wells is the first issue to be examined. Figure 2 is a dimensionless plot for the results of an analysis using the first stochastic process (case 1) of Table 1. This graph depicts the mean coefficient of variation as a function of the normalized distance from the pumping well to the ring of observation wells. Throughout this work, the distance between the pumping and observation wells is normalized by the range of the semivariogram of the stochastic process. This normalization is used only to relate the observation well location to the correlation structure of the stochastic process and does not indicate that the results are independent of the specific semivariogram range used in the analysis. Note that the confidence intervals shown on Figure 2 and following figures are approximate $95 \%$ confidence intervals calculated assuming a finite sample drawn from a normal distribution of an unknown mean and variance. Figure 2 clearly shows that the coefficient of variation increases with distance from the pumping well. This relationship is seen in all the cases examined here, and it 


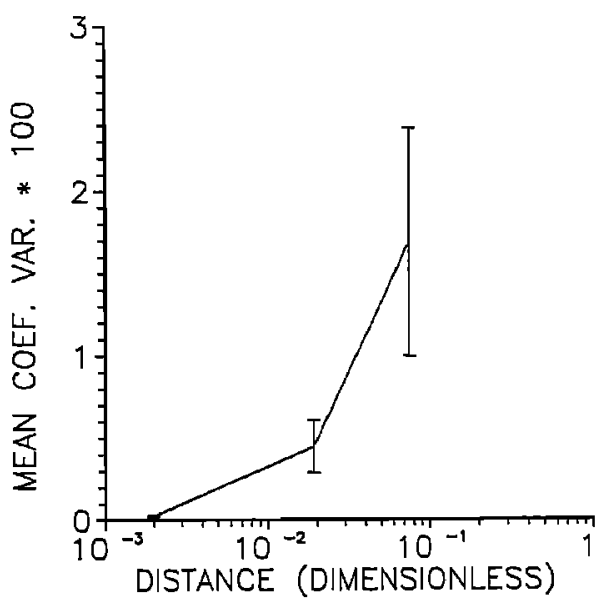

Fig. 2. Mean coefficient of variation versus distance plot for case 1 of Table 1 (distance normalized by semivariogram range); duration of analysis is three log cycles in time.

essentially reflects the greater variability in transmissivity that is being met about the circumference of the ring of observation wells as this circumference increases in size. Note that the pumping test transmissivity values employed in the analysis of angular and radial dependence are determined using the NLS procedure. The C-J procedure is only used for the analysis of temporal dependence, as is explained shortly.

Figure 3 summarizes the results of the analysis of angular dependence for cases $2-4$ of Table 1 . Clearly, as the variance of the stochastic process increases, the mean coefficient of variation also increases. These increases in the coefficient of variation are expected, as greater variability is being met over the same separation distance. The Monte Carlo simulation for case 3 is carried out for thirty realizations. Table 2 details how the statistics change with the number of realizations. The magnitude of $S \overline{C V}$ relative to $\overline{C V}$ demonstrates that the number of realizations employed in this study is reasonable, given the previously stated goals of this work. Note that the relative magnitude of $S_{C V}$ indicates that, although the estimate of the mean might be acceptable, there may be considerable uncertainty concerning the applicability of this mean value to characterize behavior in any single realization.

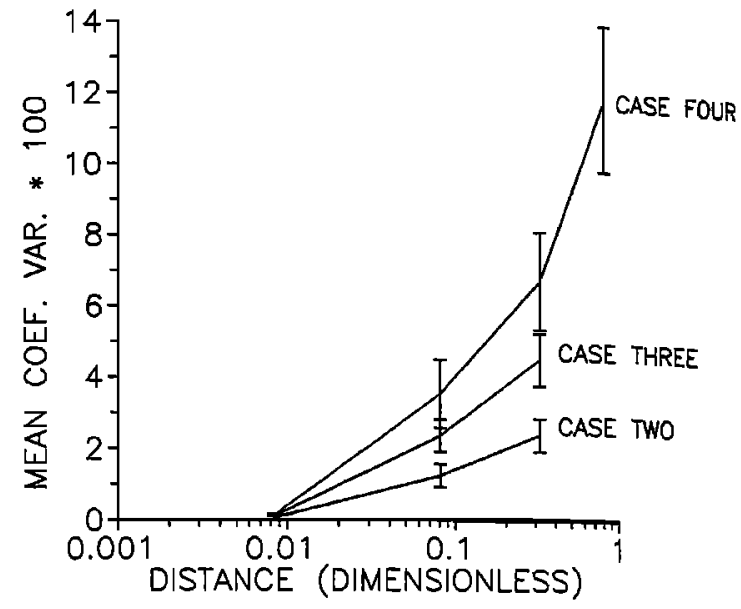

Fig. 3. Mean coefficient of variation versus distance plot for cases $2-4$ of Table 1 (distance normalized by semivariogram range duration of analysis is three log cycles in time.

For case 4 of Table $1 \mathrm{a}$ ring of observation wells is also placed at a distance of $124 \mathrm{~m}$ from the pumping well. This additional ring of wells allows behavior at distances of the order of the range of the semivariogram of the stochastic process to be examined. As shown in Figure 3, the mean coefficient of variation continues to increase through distances close to the range of the stochastic process. Although not examined further here, such increases in the mean coefficient of variation with distance would be expected to continue until the portion of the aquifer controlling the response at each observation well reaches a size at which the degree of correlation between volumes of that size begins w overwhelm the effect of the separation distance. Note that the analytical solution described by Butler and Liu [1989; An analytical solution for flow to a well in radially asymmetrical nonuniform media, submitted to Water Resources Research, 1991 (hereinafter J. Butler and W. Liu, submitted mam. script, 1991)] and Butler [1990] for flow in a radially asymmetric nonuniform aquifer can be used to study how the portion of the aquifer controlling the response at an observation well increases with radial distance from the pumpia well. On the basis of results from a study using that analytical solution (J. Butler and W. Liu, submitted manuscriph, 1991) it is clear that for a circle of observation wells at a lare

TABLE 2. Dependence of Monte Carlo Results on the Number of Realizations for Case 3 of Table 1

\begin{tabular}{ccccc}
\hline $\begin{array}{c}\text { Radius of } \\
\text { Observation, }{ }^{*} \mathrm{~m}\end{array}$ & $\begin{array}{c}\text { Number of } \\
\text { Realizations }\end{array}$ & $\overline{\mathrm{CV}} \dagger$ & $S_{C V} \ddagger$ & $S_{\overline{C V}} \S$ \\
\hline $1.3(0.008)$ & 10 & $9.91 \times 10^{-4}$ & $6.56 \times 10^{-4}$ & $.2 .07 \times 10^{-4}$ \\
& 20 & $9.50 \times 10^{-4}$ & $5.30 \times 10^{-4}$ & $1.19 \times 10^{-4}$ \\
& 30 & $9.75 \times 10^{-4}$ & $4.89 \times 10^{-4}$ & $0.89 \times 10^{-4}$ \\
$12.6(0.08)$ & 10 & $2.35 \times 10^{-2}$ & $1.48 \times 10^{-2}$ & $4.68 \times 10^{-3}$ \\
& 20 & $2.34 \times 10^{-2}$ & $1.33 \times 10^{-2}$ & $2.97 \times 10^{-3}$ \\
$49.6(0.3)$ & 30 & $2.36 \times 10^{-2}$ & $1.20 \times 10^{-2}$ & $2.19 \times 10^{-3}$ \\
& 10 & $4.36 \times 10^{-2}$ & $1.33 \times 10^{-2}$ & $4.21 \times 10^{-3}$ \\
& 20 & $4.52 \times 10^{-2}$ & $1.92 \times 10^{-2}$ & $4.29 \times 10^{-3}$ \\
& 30 & $4.51 \times 10^{-2}$ & $1.96 \times 10^{-2}$ & $3.58 \times 10^{-3}$ \\
\hline
\end{tabular}

*Normalized radius in parenthesis.

$+\overline{C V}$ is the mean coefficient of variation.

$\neq S_{C V}$ is the standard deviation of the coefficient of variation.

$\delta S \overline{C V}$ is the standard deviation of the mean coefficient of variation. 


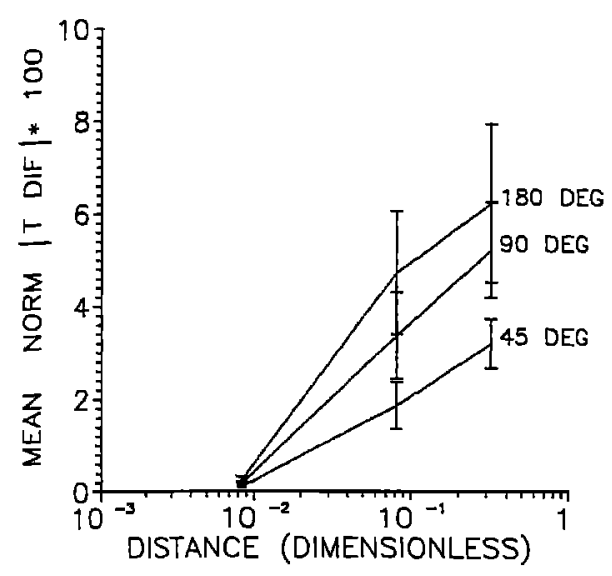

Fig. 4. Mean angular difference versus distance plot for case 4 Table 1 for three different angular separations (difference normalby the transmissivity value at the first well, distance normalized by semivariogram range, and each curve labeled according to the separation in degrees); duration of analysis is three log cyctes in time.

from the pumping well the mean coefficient of viation would be expected to approach zero in aquifers trose transmissivity variations can be represented by a second-order stationary stochastic process.

The mean normalized difference can also be employed to camenine the nature of angular dependence. Figure 4 depicts thean normalized difference in the angular direction as a bection of distance for case 4 of Table 1 . Results for three spacings are displayed. The increases with angular pacing seen up to at least $90^{\circ}$ are as expected, since greater variability in transmissivity is being met at greater separation weances.

This examination of angular dependence has primarily focused on the estimation of means taken over a stochastic process. In order to quantify the error that might be incurred by using these mean values to characterize behavior in a single realization, the variability of the coefficient of variaacross the realizations is examined. Standard deviations of the coefficients of variation are estimated to characterize this variability and are given in Table 3 . In no case is a stendard deviation greater than $50 \%$ of the mean observed for wells at $49.6 \mathrm{~m}$.

Histogram plots of the coefficients of variation are also constructed. A representative plot from this group is given in Feure 5. Although the plots are decidedly asymmetric in all

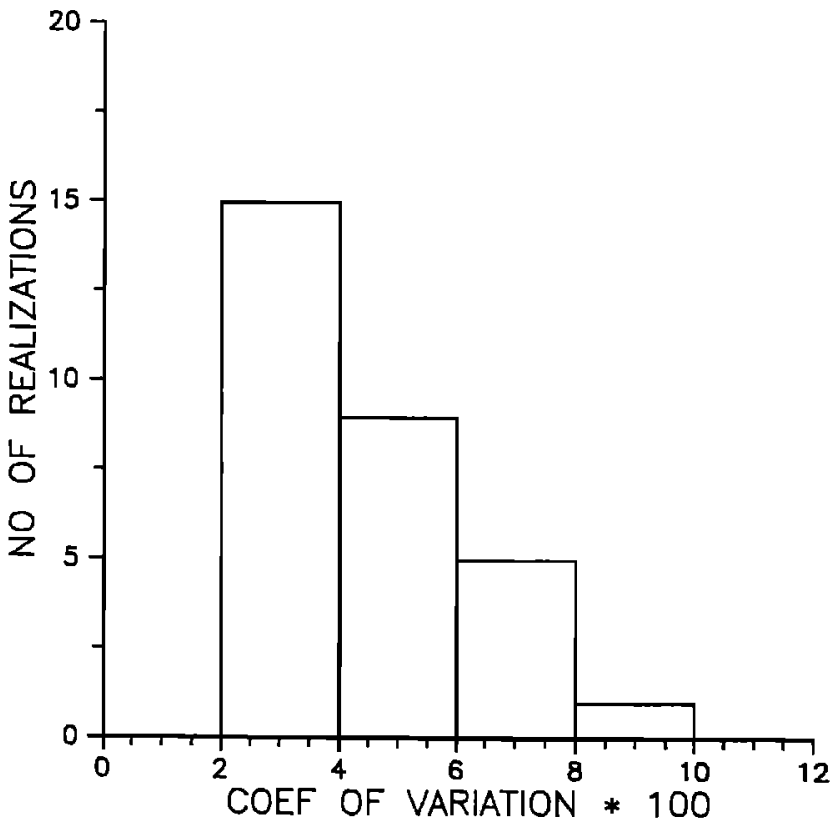

Fig. 5. Histogram of the coefficient of variation within a realization for case 3 of Table 1 using observations wells at $49.6 \mathrm{~m}$; duration of analysis is three log cycles in time.

cases, no distant outlier values are observed, giving credence to the assumption that the sample histograms reasonably represent the form of the population distributions. Given the relative and absolute magnitude of the variability across the realizations, the mean values determined in this analysis should be reasonable estimates of behavior in a single realization.

In summary, the results of the analysis of angular dependence show that the variability of pumping test transmissivity values calculated about the circumference of a circle of observation wells centered on the pumping well increases with the variance of the stochastic process and the distance between the pumping and observation wells. In none of the examined cases, however, does the magnitude of the variability reach a level of much concern for practical applications. Although the significance of the magnitude of the coefficient of variation or the mean normalized difference is a function of the specific use for which the transmissivity estimates will be applied, a coefficient of variation and a normalized difference of less than 0.1 can certainly be assumed negligible for most practical applications.

TABLE 3. Sample Means and Standard Deviations of Functions Used to Characterize the Dependence of Pumping Test Transmissivity on Angular and Radial Position of Observations

\begin{tabular}{cccccc}
\hline & \multicolumn{2}{c}{ Angular Analysis } & & \multicolumn{2}{c}{ Radial Analysis } \\
\cline { 2 - 3 } \cline { 5 - 6 } Case Number & $\overline{C V}$ & $S_{C V}$ & & $\overline{A N D^{*}}$ & $S_{A N D^{\dagger}}$ \\
\hline 1 & $1.56 \times 10^{-2}$ & $7.66 \times 10^{-3}$ & & $2.22 \times 10^{-2}$ & $7.55 \times 10^{-3}$ \\
2 & $2.39 \times 10^{-2}$ & $9.67 \times 10^{-3}$ & & $9.32 \times 10^{-2}$ & $6.71 \times 10^{-2}$ \\
3 & $4.51 \times 10^{-2}$ & $1.96 \times 10^{-2}$ & & $\mathrm{NC}$ & $\mathrm{NC}$ \\
4 & $6.73 \times 10^{-2}$ & $2.95 \times 10^{-2}$ & & $3.06 \times 10^{-1}$ & $2.53 \times 10^{-1}$ \\
\hline
\end{tabular}

Both analyses have their observation points at $49.6 \mathrm{~m}$; the nonlinear least squares analyses are performed over an interval of approximately three log cycles in time. $\mathrm{NC}$, not computed (radial analysis not carried out for this case).

"AND is the mean of the average normalized difference within a realization.

$\uparrow S_{A N D}$ is the standard deviation of the average normalized difference within a realization. 


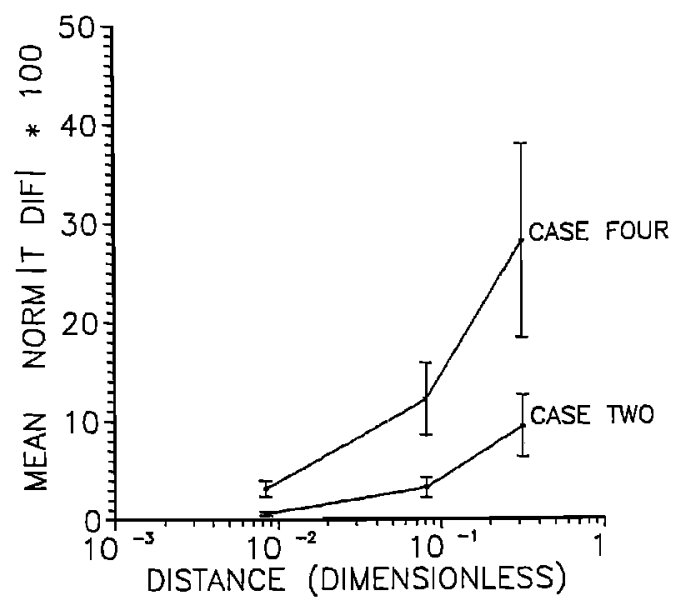

Fig. 6. Mean radial difference versus distance plot for cases 2 and 4 of Table 1 (difference normalized by the transmissivity value at the pumping well and distance normalized by the semivariogram range); duration of analysis is three log cycles in time.

\section{Dependence on Radial Location}

The dependence of pumping test transmissivity on the radial location of observation wells is the next issue to be examined. As discussed earlier, the normalized absolute value of the difference in transmissivity between that calculated at an observation well and that at the pumping well is the quantity employed to characterize radial dependence. Figure 6 depicts the radial dependence relationships observed for cases 2 and 4 of Table 1 . The relationships are similar to those illustrated in Figure 3, with increases in the variance of the stochastic process and the distance to the observation well leading to a larger mean difference. Although the general form of the relationships is similar to that observed in the angular analysis, the magnitude of the dependence is considerably greater. If, as in the angular analysis, a mean normalized difference of less than 0.1 can be assumed negligible, Figure 6 indicates that the difference between a transmissivity value calculated at the pumping well and one calculated at a distant observation well may be significant in the more variable aquifers. Unlike the angular case, this difference should not decrease to zero at a very large distance from the pumping well. Although not demonstrated here, theoretical considerations indicate that the difference should stabilize near the mean value of the following quantity [see Butler and Liu, 1989, also submitted manuscript, 1991]:

$$
\left|1-\frac{T_{g m}}{T_{p w}}\right|
$$

where $T_{g m}$ is the geometric mean of the transmissivities employed in the realization, and $T_{p w}$ is the transmissivity calculated from drawdown at the pumping well. The geometric mean is used in (S) as a surrogate for the value toward which the transmissivity of increasingly larger volumes of the aquifer will converge as a result of the methodology employed in this study, an issue that is discussed further in succeeding pages.

The analysis of radial dependence is incomplete without considering the error that may be introduced when employing an estimate of the mean radial dependence to character- ize behavior in a single realization. The sample standan deviations, employed to quantify the variation in the normat ized difference across the sampled realizations, are listed i Table 3. Note that standard deviations greater than $70 \%$ of the mean are calculated for cases 2 and 4 . Such large relative values coupled with the magnitude of the estimated mean imply that there is considerable uncertainty when estimatim behavior in a single realization using the mean relationships. Histogram plots of the absolute normalized difference with a realization reinforce this point. For case 4 the mean over the sampled realizations is shown to have relatively limited value for characterizing behavior in a single realization when considering observations at $49.6 \mathrm{~m}$ (Figure 7 , line A). Thx average difference from one realization indicates that at leas an order of magnitude change in transmissivity is possible over that separation distance. Although the average normat ized differences are smaller closer to the pumping wed (Figure 7, line B) or for a less variable process at the same radial distance (Figure 7 , line $C$ ), even in these instances. differences of over 3 times the mean value are seen. Thus the mean values must be used cautiously when characterixing the radial dependence in any single realization. Note that the form of the histogram given by line $A$ of Figure 7 indicates that more realizations would be required in the Monte Carb simulation to better estimate the nature of the distribution of the average normalized difference in the more variable cases.

In summary, the results of the above analysis show the for case 4 the issue of radial dependence is of concern for distances greater than about $15 \mathrm{~m}$. For case 2, however, the issue of radial dependence is only a concern at much larger distances, although considerable variations are possible at distances as small as $50 \mathrm{~m}$. Note that a much greater variability across realizations is seen in the radial analysis than in the angular. This is due to small areas of anomalous character in the vicinity of the pumping well having a

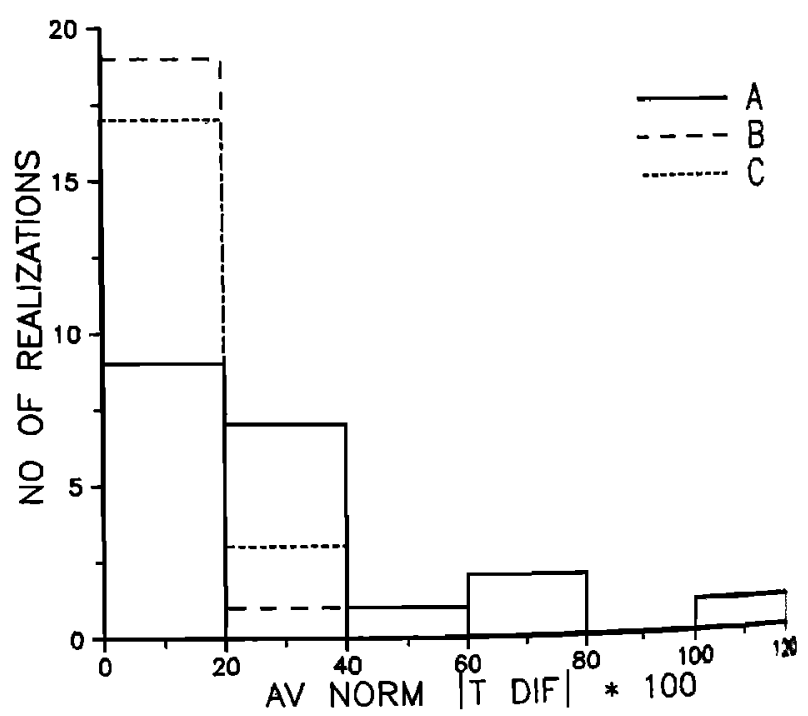

Fig. 7. Histograms of the average radial difference with 1 realization for a duration of analysis of three log cycles in tion (difference normalized by the transmissivity value at the pumpus well; line A, results for case 4 of Table 1 using observation weth $49.6 \mathrm{~m}$; line $\mathrm{B}$, results for case 4 of Table 1 using observation $4.6 \mathrm{~m}$; line $B$, results for case 2 of Table 1 using observats wells at $49.6 \mathrm{~m}$ ). 


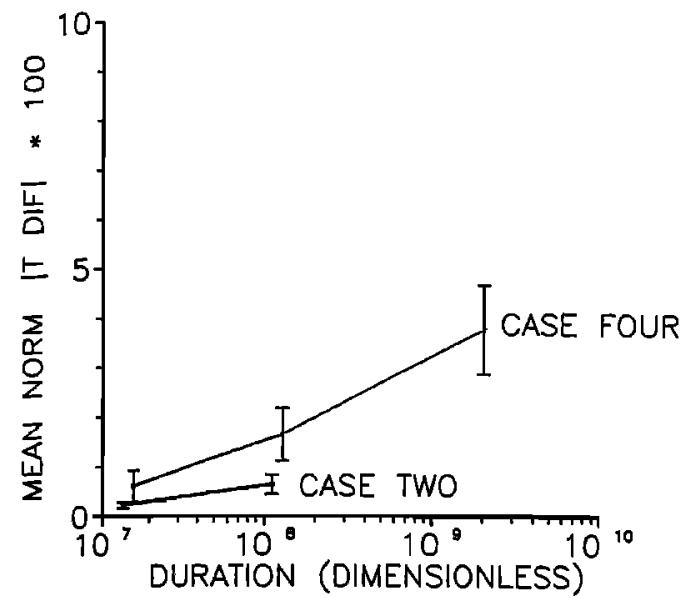

Fig. 8. Mean temporal difference versus duration of analysis for ases 2 and 4 of Table 1 (difference normalized by the transmissivity calculared over one log cycle in time and duration normalized as siscussed in text); observation point is the pumping well.

considerable influence on the radial dependence as a result of their large impact on drawdown at or near the pumping well. Such small anomalous areas have much less influence drawdown when located near observation wells placed long the circumference of a circle at a distance from the pumping well. This radial dependence in the impact of small areas of anomalous properties on drawdown, which is considered further by Butler and Liu [1989, also submitted manuscript, 1991], results in greater variability being seen across the realizations for the radial analysis.

\section{Dependence on Temporal Location}

The issue of temporal dependence can only be partially dressed using the simulation results, owing to discretization effects propagating into the transmissivity calculations and producing sizable uncertainties at small dimensionless times, as discussed in Appendix C. Therefore only the temporal dependence at the pumping well is considered bere. The normalized absolute difference described earlier is employed to characterize temporal variations in transmissivity. In this case the transmissivity calculated over the first log cycle in time serves as the normalizing quantity.

Figure 8 is a dimensionless plot of the mean normalized absolute transmissivity difference versus the duration of the alysis. This plot shows that the mean difference does not exceed 5\% for either case. Thus for observation points near the pumping well the calculated transmissivity is essentially independent of the duration over which the analysis is performed. Note that the analysis is carried out for a larger duration for case 4 , owing to the longer pumping period used inat case. Note also that the duration of the analysis is potted as a dimensionless quantity $\left(4 T_{E V} t / S r^{2}\right)$, where $T_{E V}$ is the expected value of the stochastic process.

$U_{p}$ to this point, only pumping test transmissivities calcuued using the nonlinear least squares routine have been employed. Often, however, the Cooper-Jacob approach is for analysis of pumping-induced drawdown. Though two methods produce identical results in uniform units atmensionless times greater than 100 , the question of their equality in nonuniform units needs to be addressed. This of equality is evaluated here by comparing results from

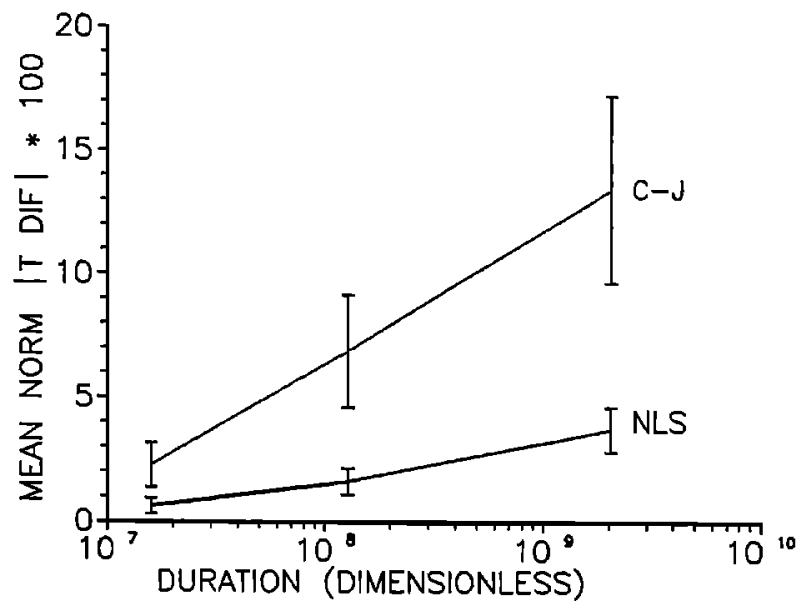

Fig. 9. Mean temporal difference versus duration of analysis for case 4 of Table 1 (difference normalized by the transmissivity calculated over one log cycle in time, duration normalized as discussed in text, and curves labeled according to the method of drawdown analysis); observation point is the pumping well.

the two approaches using drawdown at the pumping well, where dimensionless times are much greater than the suggested limiting value of 100 .

The temporal dependence of transmissivity values calculated using the NLS and C-J approaches is illustrated in Figure 9 for case 4. Though, clearly, the transmissivities calculated using the C-J approach display a greater temporal dependence, the magnitude of the mean normalized difference does not merit a great deal of concern. An analysis of the variability of the normalized difference across the sampled realizations, however, indicates that temporal dependence may be important in the C-J case for the more variable aquifers. This is shown by the histogram plot of Figure 10, where normalized differences of greater than 0.30 are observed for the C-J estimates. Variability across the sampled realizations is not important for the NLS estimates. Although not demonstrated here, theoretical considerations

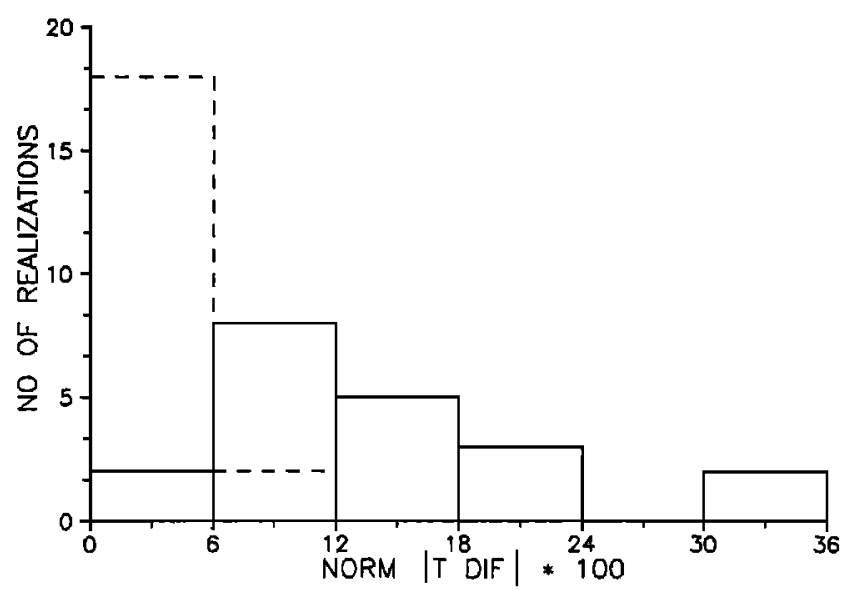

Fig. 10. Histograms of the temporal difference within a realization for case 4 of Table 1 for a duration of analysis of four log cycles in time (difference normalized by the transmissivity calculated over one log cycle in time: solid line, results for transmissivity calculated using the Cooper-Jacob approach and dashed line, results for transmissivity calculated using the nonlinear least squares approach); observation point is the pumping well. 


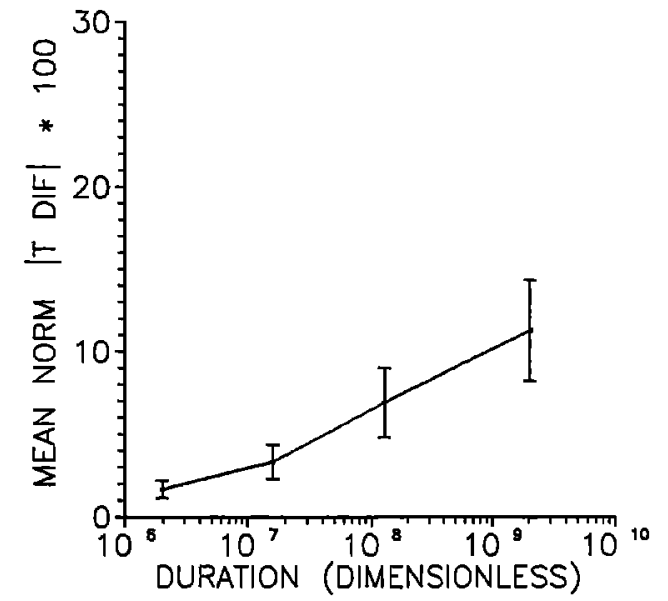

Fig. 11. Mean difference between transmissivities calculated using Cooper-Jacob and nonlinear least squares methodologies versus duration of analysis for case 4 of Table 1 (difference normalized by the nonlinear least squares transmissivity value and duration normalized as discussed in the text); observation point is the pumping well.

indicate that the C-J plot of Figure 9 should eventually stabilize at a level near that given in (5) (see J. Butler and W. Liu, submitted manuscript, 1991).

The actual difference between the NLS and C-J transmissivities is shown in Figure 11, a plot of the mean normalized absolute transmissivity difference versus the duration of the analysis. The NLS transmissivity serves as the normalizing quantity for this plot. Figure 11 indicates that the normalized difference between the NLS and C-J parameters increases with the duration of the analysis. This difference is solely a function of aquifer nonuniformity, since experiments with the same numerical model produced identical transmissivity estimates from NLS and C-J analyses in a uniform aquifer. Butler [1990] provides a lengthy discussion of the basis for the difference in parameter estimates produced by these two analytical techniques. Essentially, these two procedures provide dissimilar estimates in nonuniform aquifers because of their emphasis on properties of material in different portions of the aquifer. This difference in emphasis arises from the fact that the NLS procedure involves the fitting of a theoretical drawdown versus time curve to the data, while the C-J procedure, as conventionally performed, simply involves fitting a straight line to data on a semilog plot. The result is that the NLS approach places a heavier emphasis on the transmissivity of material in the vicinity of the observation well, while the $\mathrm{C}-\mathrm{J}$ approach emphasizes the transmissivity of material in the front of the cone depression (the leading edge of the cone of depression from which the majority of the pumped water originates). As discussed by Butler [1990], one consequence of this difference in emphasis is that the NLS procedure is more appropriate for parameters used to estimate pumping-induced drawdown, while the C-J procedure is more appropriate for parameters used to estimate aquifer yield. Another consequence of this difference is that parameters estimated from the NLS approach display a greater angular and radial dependence, while the C-J parameters display a greater temporal dependence. It should be noted that the relationships depicted in Figure 11 pertain to observation wells located near the pumping well. As Butler [1990] shows, the difference between the NLS and

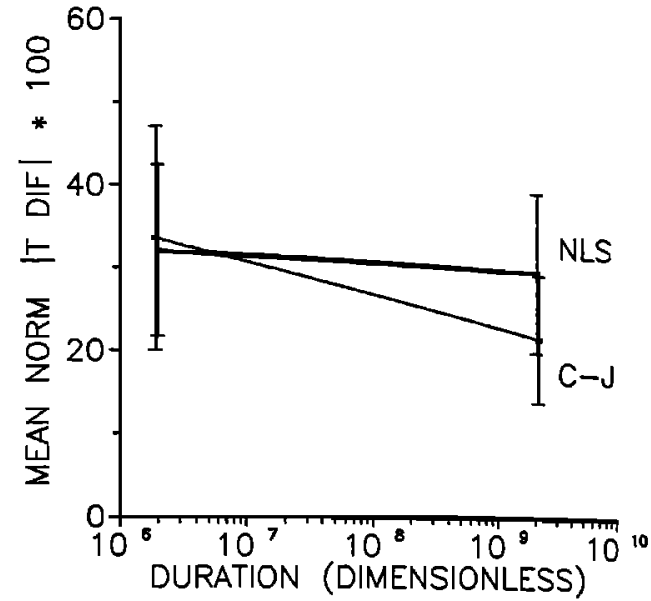

Fig. 12. Mean difference between pumping test transmissivities and the geometric mean of the realization versus duration of analysis for case 4 of Table 1 (difference normalized by the geometric mean. duration normalized as discussed in the text, and labels as defined the text); observation point is the pumping well. Note that the C.J plot has been artificially offset a small amount in time in order to distinguish the difference in confidence intervals.

C-J parameters will decrease with increases in the distance between the observation and pumping wells.

\section{Estimation of Regional Transmissivity}

An important use of transmissivities estimated from a pumping test is as inputs into regional flow models, with the test estimates being employed to characterize the transmissivity over an area of considerable extent. The results of the temporal dependence analysis of case 4 can be employed to briefly assess the viability of using pumping test transmissivities to characterize regional properties. The geometric mean of the realization is used here to represent the regional transmissivity as a result of the methodology employed in this study. As described in Appendix A, the geometric mean is used in this work to combine the block transmissivities produced by the turning bands generation into effective transmissivities for elements of the flow model. At a very large distance from the pumping well, the increasing size of the elements of the flow model results in the convergence of the element transmissivity on the geometric mean of the realization. Thus if a pumping test transmissivity is a reasonable representation of the property at the regional scale. the test estimate should converge on the geometric mean of the realization with increases in the duration of the analysis.

Figure 12 is a plot of the mean absolute normalized difference between the pumping test transmissivities and the geometric mean of the realization versus duration of the pumping test analysis. The geometric mean of the realization serves as the normalizing quantity in this plot. Note that the C-J transmissivity appears to approach the geometric mean much faster than the NLS parameter. The apparent convergence of C-J transmissivities on the geometric mean of the realization, coupled with the assertion that the C-J approach emphasizes the material in the front of the cone of depression [Butler, 1988, 1990], suggests that a possible approach for estimating transmissivity on a regional scale would be to analyze the drawdown data over a single log cycle at larper times of pumpage (assuming boundary effects are negligible). 


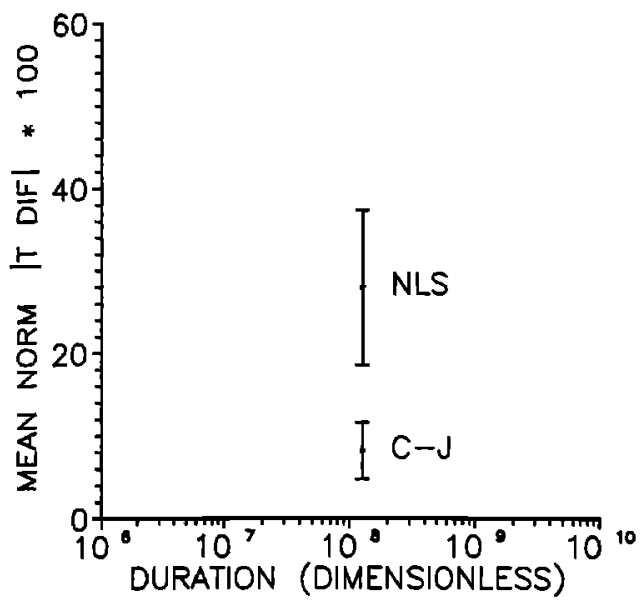

Fis. 13. Mean difference between the pumping test transmissivFies and the geometric mean of the realization at a large duration of masis for case 4 of Table 1 (difference normalized by the geometic mean, duration normalized as discussed in the text, and labels as ined the text); observation point is the pumping well.

Fure 13 displays the markedly better estimates achieved trough this approach and the analogous results produced wing the NLS methodology. The C-J approach applied at a arge dimensionless time thus appears to yield a viable epproximation of the regional transmissivity. This result is in reeping with an earlier suggestion [Toth, 1967] that late time malysis of the drawdown record using the Cooper-Jacob methodology would be a reasonable approach for evaluation of regional aquifer properties.

An analysis of the variation of the normalized difference cross the sampled realizations is useful to assess the general applicability of the findings of the previous paragraph. Line A of Figure 14 is a histogram plot depicting the spread of vabes corresponding to the leftmost point of Figure 12, valid for both the NLS and C-J analyses. This spread does not decrease substantially with increases in either duration or to isitial time of a NLS analysis. For a C-J analysis, wowever, a notable decrease in the variability across realizations is seen with increases in both the duration and the inial time of the analysis. Line B of Figure 14 illustrates the spread corresponding to the rightmost point of Figure 12 for a $\mathrm{C}-\mathrm{J}$ analysis, while line $\mathrm{C}$ displays the dramatic decreases observed when a C-J analysis is carried out over a single log cycle at a large time of pumpage. The results of this analysis of the variation across the sampled realizations thus support the conchusion of the previous paragraph that a C-J analysis a a large dimensionless time would be a reasonable apmach for estimation of transmissivity on a regional scale. It chould be noted that, as with Figures $9-11$, these results pertain to observation wells located near the pumping well. The NLS methodology applied to pumping-induced drawdoma at a distant observation well should produce a better ate of the regional transmissivity than that given by the NLS results in Figures 12 and 13.

\section{SUMmary AND CONCLUSIONS}

This article has examined the dependence of the transmisivily estimated from a pumping test in a nonuniform aquifer the spetial and temporal location of observations and the method of drawdown analysis. The results of this work can be summarized as follows:

1. For the network of observation wells employed here the variability of pumping test transmissivity values calculated about the circumference of a circle of observation wells centered on the pumping well increases with the variance of the stochastic process and the distance between the pumping and observation wells. The magnitude of the variability, however, never reaches a level of practical concern in any of the cases examined here. Although this conclusion concerning angular dependence is based on the mean behavior over the sampled realizations, it is considered a reasonable description of conditions within a single realization, since the variability over the sampled realizations is small. In addition, theoretical considerations and the analytical results discussed by Butler [1990] indicate that the angular variations should go to zero at a large distance from the pumping well for aquifers of the type considered here. Therefore in such aquifers a transmissivity value calculated from drawdown at a single well should adequately characterize the property in the angular direction for most practical applications.

2. The variability of pumping test transmissivity values in the radial direction also increases with the variance of the stochastic process and the radial distance from the pumping well. Unlike the angular dependence, however, the radial dependence is large enough to be of concern for practical applications. In addition, the variability over the sampled realizations is large enough to cause the mean estimates to be of limited value at large distances from the pumping well. Transmissivity values calculated using drawdown from distant observation wells must therefore be used with caution to characterize conditions close to the pumping well.

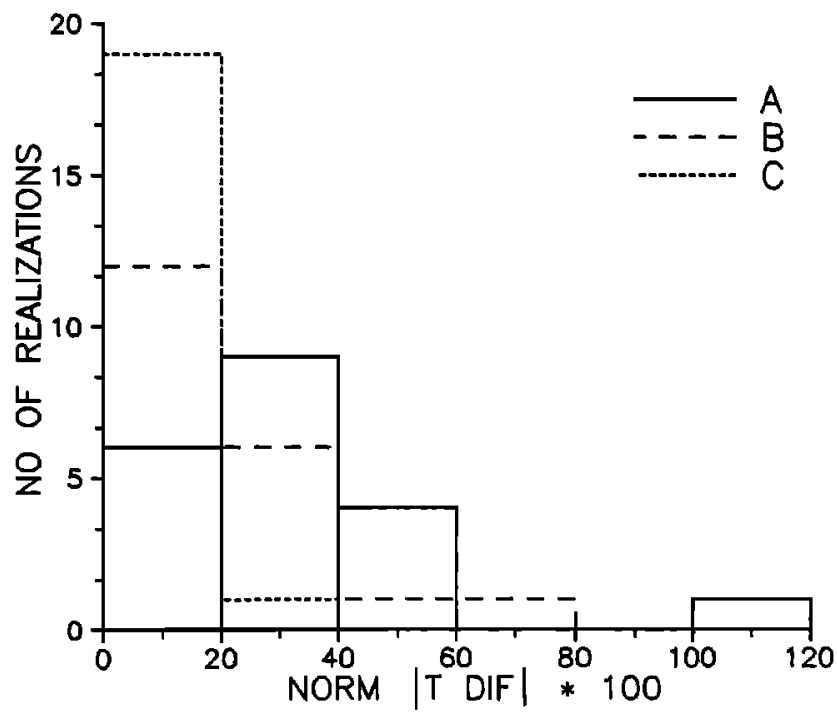

Fig. 14. Histograms of the difference within a realization between the pumping test transmissivities and the geometric mean of the realization for case 4 of Table 1 (difference normalized by the geometric mean; line A, results for leftmost position of Figure 12, valid for both C-J and NLS analyses, where duration of analysis is one log cycle in time; line B, results for rightmost position of Figure 12 , valid only for C-J analysis, where duration of analysis is four $\mathrm{log}$ cycles in time; and line C, results for C-J analysis of Figure 13, where duration of analysis is one log cycle in time); observation point is the pumping well. 
3. Transmissivity values calculated from observation points near the pumping well have a relatively weak dependence on the duration of the drawdown analysis. This dependence is a function of the specific methodology employed in the analysis. Variability over the sampled realizations is large enough in the case of the Cooper-Jacob methodology to merit some concern when employing the results obtained from a test of a short duration to characterize aquifer response to pumpage of a much greater duration, even in the absence of boundary effects. Although not directly examined here, Figures 12 and 13 indicate that parameter dependence on the initial time of the analysis is much greater than the dependence on the total duration of pumpage for the Cooper-Jacob approach.

4. Analytical approaches that are equivalent in uniform aquifers yield differing parameters in nonuniform units as a result of different data-fitting procedures. This difference in fitting procedures causes the nonlinear least squares and Cooper-Jacob approaches to heavily weight properties of material in different portions of the aquifer. As Butler [1990] discusses, the superiority of one approach over the other depends upon the specific use of the parameters (e.g., drawdown predictions versus yield predictions). Note that the magnitude of this difference decreases with distance from the pumping well.

5. A transmissivity value obtained from a Cooper-Jacob analysis applied at large dimensionless times is, given that boundary effects are negligible, a reasonable representation of the transmissivity on the regional scale. The viability of a NLS analysis for characterization of regional properties is dependent on the distance of the observation well from the pumping well.

The general conclusion resulting from this work is that the conventional approaches for the analysis of pumpinginduced drawdown, for the most part, appear viable in nonuniform aquifers of the type considered here. Viable is used here in the sense that drawdown at one observation well can be used to calculate a transmissivity value that is a reasonable estimate for most practical applications. Clearly, however, the dependence of pumping test transmissivity on the spatial and temporal location of observations, and the method of drawdown analysis, must be understood in order that the design, performance, and analysis of a pumping test be directed toward answering the questions of most relevance for the particular application.

Clearly, the results and conclusions of this work must be viewed within the context of the assumptions that are employed in the research. Further work is required to remove some of these assumptions and increase the generality of the analysis. The most important limitations arising from the assumptions employed here are the following:

1. The results are only applicable to aquifers with a considerable degree of horizontal continuity, since the semivariogram ranges employed here are quite large. Although it would be instructive to extend this analysis to systems with considerably smaller ranges, the results of this work do give a clue as to what might be expected in such systems. Comparison of cases 1 and 2 of this study indicates that the angular dependence should change relatively little while the radial dependence should increase greatly, over the distances considered here, with a decrease in the semivariogram range. The distance at which the angular dependence becomes negligible should also decrease. Similarly, radial and temporal dependence relationships should stabilize net the level given in (5) at smaller distances and times, respoc. tively. If the semivariogram range is very small (on the order of a few meters), pumping test transmissivity should disphy only a very weak dependence on spatial and tempon location of observations, and $T_{p w}$ of (5) should be approximately equal to $T_{g m}$. Thus the worst case condition shouk be an aquifer with a moderate degree of horizontal contin (i.e., ranges of the order of tens of meters).

2. This analysis only considers the impact of variation in the transmissive properties of an aquifer on pumping transmissivity. Additional work is required in order to assess the impact of variations in the storage properties of aquifer on pumping test parameters. A major issue in such extension would be the definition of the cross-correlatio relationships between the storage and transmissive properties of a unit.

3. The results of this work are limited to aquifers whose transmissivity variations can be represented by a specific class of stochastic processes. These stochastic processes are composed of random variables (ln $K$ ) characterized by normal marginal probability density functions (pdfs) with first- and second-order moments that are stationary in spact and coefficients of variation that are relatively small. $h$ addition, the correlation between the random variables be represented by isotropic semivariograms. Further wort is necessary to extend the analysis to units that can be repre sented by nonstationary marginal pdfs, marginal pdfs with large coefficients of variation, and/or anisotropic semivariograms. Systems with large coefficients of variation and anisotropic correlation, such as systems of sand and chy lenses in alluvial aquifers, would be expected to have significantly different dependence relationships than those observed here. The analytical solution developed by Butier and Liu [1991] for the case of pumping-induced drawdown the vicinity of a strip of one material embedded in a matrix $x$ differing properties can provide some information concen ing the dependence relationships that would be expected such systems. Note that this analytical solution also id cates that temporal dependence in parameter values could be very large in units that can be represented by nonstationary marginal pdfs.

4. The analyses of this work are limited to pumping tests in confined flow systems or in unconfined systems in which the pumping-induced drawdown is small relative to the tow saturated thickness. Considerable additional work would be required for the analysis of pumping tests in unconfined systems in which pumping-induced drawdown is large reta tive to the total saturated thickness. A three-dimension representation of the property variations would be necessan? in the unconfined case since the vertical variations in $b$ draulic conductivity within the aquifer cannot be ignored as in the confined case [Javandel and Witherspoon, 1969. Butler, 1986].

Finally, it must be emphasized that the research described in this article focuses on the viability of conventional pat ing test analysis methodology in nonuniform aquifers. Lith direct attention is given to the issue of how the results fro pumping test analyses can be used to characterize the nature of aquifer nonuniformities. The definition of observatio well networks and pumping strategies to help define nature of property variations over an area are the subject ongoing work. 


\section{APPENDIX A}

An estimate of the dependence of pumping test transmissivity on spatial and temporal location of observations is obtained in this work by using Monte Carlo simulation. One of the primary attractions of this methodology lies in its deplicity. Realizations of a stochastic process representing transmissivity variations within the modeled area are cerated, and the deterministic flow equation is solved over ach realization. The procedure is repeated until the summy output statistics are considered an acceptable approxmetion of the parameters of the output stochastic process. Whin this process of repetitive generation and solution lie major issues associated with Monte Carlo simulation: (1) criteria for terminating the procedure, and (2) the large mer of computations necessary to achieve an acceptable sobution. This appendix briefly describes how these issues we addressed in this work.

The standard approach for terminating Monte Carlo simtheion is to evaluate the estimates of the moments of the atput stochastic process. When the standard deviations of these estimates decrease to a level defined as acceptable, the mocedure is terminated. The dependence of the standard deviations on the inverse square root of the number of ralizations often results in a large number of realizations being required in order to achieve an acceptable level of tror. A major thrust of research on Monte Carlo simulation mos involved the development of realization (or variance) reduction techniques, methods designed to reduce the number of realizations required to achieve a certain level of error [Hommersley and Handscomb, 1964; Rubinstein, 1981]. Stratified sampling is the realization-reduction technique moyed in this work. This approach involves the subdivison a probability density function describing some charceristic of realizations of the input stochastic process (inpu pdf) into disjoint intervals and then the selection (radom or otherwise) of a certain number of samples from ach interval. This method forces the sampling of values in of the intervals and thus relatively rapidly approximates the input pdf.

The Monte Carlo simulation approach employed here cophasizes the utilization of a stratified-sampling scheme, in cajunction with a method for approximating the sampled iput pdf, in order to greatly reduce both the realizationparation and the flow simulation computations. Essenthe approach can be divided into the following three steps:

1. The form of the input pdf that is to be sampled by the matifed-sampling scheme is determined. For this work the sampled quantity is the variance of the transmissivities within a realization. This variance is considered to succiactly characterize the variability of transmissivity within a realization. The form of the pdf for the realization variances is approximated by repeated generation of realizations until the intervals of the pdf can be reasonably estimated. One realizations is considered a sufficient number for work. Since, in a pumping test, the significance of moperty variations decreases with distance from the pumpwell [MCElwee and Yukler, 1978; Butler, 1990], the above cheme is modified so that each of the generated realizations cocompasses a portion of the circular ( $1930 \mathrm{~m}$ in radius) under used in the pumping test simulations. The portion here is the 200 by $200 \mathrm{~m}$ square centered on the pumping well. The error introduced by considering the variability within $100 \mathrm{~m}$ of the pumping well (the near-well variance) as playing the major role in determining the dependence relationships is assumed minimal for the aquifer and observation well configurations of this work. Computational reductions achieved by using this assumption are significant.

2. The pdf of the near-well variance is then subdivided into disjoint intervals of approximately equal probability. Ten intervals are used here, based on the deciles of the generated pdf. Variance values are selected randomly from each interval. Realizations of the entire aquifer with the selected near-well variances are then generated. It is important to note that, although the selection of realizations is based on the near-well variance, the realizations generated for the flow simulations cover the entire aquifer. The variance of the portion of the generated realizations at a distance from the pumping well is just not considered in the sampling process. This variation, however, is included in the realizations.

3. Each of the generated realizations is then input into the finite element flow model. A pumping test is simulated, and the computed drawdown at the observation wells is analyzed using one of the procedures described in the text. The mean and standard deviation of the various functions employed to characterize spatial and temporal dependence over the sampled realizations are the statistics of primary interest. It is assumed that the mean is approximately normally distributed, at least in the central portions of its distribution, by invoking the central limit theorem [Huntsberger and Billingsley, 1973]. This assumption allows confidence intervals about the expected value of the various functions to be calculated. These confidence intervals are then employed as part of the criteria to determine whether the Monte Carlo simulation should be terminated.

The convergence criteria employed to terminate the Monte Carlo procedure, although based on the confidence intervals described above, are rather subjective in nature. These criteria are the width of the confidence intervals, the clarity of the studied relationships, and the magnitude of the involved quantities. For example, consider the plot of Figure 2. Even though the confidence intervals are relatively wide, termination of the computations occurs because the distance dependence of the coefficient of variation is clearly displayed and the magnitude of the coefficient of variation is small. In this work the convergence criteria are considered met in most cases after two rounds of stratified sampling (one sample per interval per round) from the input pdf of the near-well transmissivity variance. Note that several rounds of stratified sampling allow the variations both between and within intervals of the input pdf to be sampled, thus facilitating convergence.

As noted in the text, a realization of transmissivities is first generated in a Cartesian coordinate system. This output is then transformed into the polar coordinate system used in the pumping test simulations. Essentially, the polar coordinate grid of elements is lain directly over the grid of rectangular blocks generated by the TUBA program. The portion of each rectangular block lying within an element of the flow model is calculated, and element transmissivities are computed as area-weighted geometric averages of the block contributions. Note that the use of the geometric mean to compute the effective transmissivities of the elements of 
the flow model should be viewed as a measure of convenience. Further work is required to actually define the appropriate form for effective transmissivity in transient simulations.

\section{APPENDIX B}

The Quadra Sands data set of Smith [1978] is the basis of the stochastic processes employed in this study. The portion of the data set used here consists of hydraulic conductivity measurements taken on repacked cores collected every 0.3 m during vertical and horizontal traverses along the outcrop of the formation. Both traverses were $30 \mathrm{~m}$ in length, resulting in 100 cores being collected in each direction. The bedding plane was subhorizontal so that the vertical and horizontal traverses are considered to be perpendicular and parallel to bedding, respectively. A considerable amount of data processing is required before the traverse data are in a form that allows the definition of a stochastic process that can be used in the Monte Carlo simulation. This appendix briefly outlines the major steps in this processing effort.

Since this data set is to be used to characterize the variability in the transmissive properties of a unit, the first step is to quantify that variability. The semivariogram [Journel and Huijbregts, 1978] is chosen here as the statistic to quantify the degree of correlation between property values at different points within a unit. Theoretical exponential semivariogram models of the following form are fit to the experimental data:

$$
\gamma(\mathbf{r})=\sigma^{2}\left(1-e^{-r / a}\right)
$$

where $\gamma(\mathbf{r})$ is the semivariogram value for separation vector $r, \sigma^{2}$ is the sill (variance), $a$ is the normalizing parameter (equal to range/3), and the range is the distance beyond which correlation can be considered essentially zero. The two semivariograms resulting from the fitting of the vertical and horizontal traverse data are

$$
\begin{gathered}
\gamma_{v}(\mathbf{r})=0.400+0.297\left(1-e^{-r / 1.33}\right) \\
\gamma_{h}(\mathbf{r})=0.0394\left(1-e^{-r / 0.9}\right)
\end{gathered}
$$

where $\gamma_{v}(\mathbf{r}), \gamma_{h}(\mathbf{r})$ are semivariograms based on data from the vertical and horizontal traverses, respectively. Note that a nugget effect [Journel and Huijbregts, 1978] has been included in (B2) in order to consider the large variations at a scale smaller than the sampling interval.

As shown by (B2) and (B3), the variance in the vertical direction is significantly greater than that in the horizontal over the scale of measurement. This is to be expected since data from a sampling traverse perpendicular to bedding should generally display much greater variability than data from a traverse of equal length in the bedding plane. This occurs because the traverse perpendicular to bedding crosses a significantly greater number of beds. For purposes of this work, property variations are divided into those observable over the scale of a single bed (intrabed variations) and those observable over a collection of beds (interbed variations). Equation (B2) is assumed to be a reasonable representation of the variations over the interbed scale within the Quadra Sand. Given the much smaller variation seen in the horizontal traverse, it is assumed that (B3) is a representation of the variations over the intrabed scale. A fundamental question therefore is how to represent the variability in the horizontal direction at an interbed scale. It is assumed here that the variance in the vertical directim would be reproduced in the bedding plane if a large enou separation distance is employed. This is somewhat anatogous to Walter's law of sedimentology [Miall, 1984], which states that lateral facies relationships will be reproduced ina vertical section.

Given the variance (sill plus nugget) of the vertical traverse, the question then becomes, what is the form and range of the interbed horizontal semivariogram? An exponential form is chosen here to model the nested structure that would be expected on the horizontal interbed scale [ $d$ Marsily, 1984]. It is assumed that the nested structure consists of a series of intrabed semivariograms, each with the same variance. Given the interbed sill, (B1) can be used to calculate the interbed range if the relationship between $y$ and $\mathbf{r}$ is known at one point on the interbed semivariogran The approach used here is to set $r$ equal to an estimate of the average length of a bed and set $\gamma(r)$ equal to the intrabed variance, thus forcing the interbed semivariogram to roughly approximate the variability observed on the intrabed scale. An average bed length of $12 \mathrm{~m}$ is used, based on the experimental horizontal semivariogram of the Quadra Say data, which shows no indication of a bed boundary prior to that separation distance [see Butler, 1986]. At larger separtion intervals the experimental semivariogram is more difficult to interpret because of the considerable fluctuations arising as a result of the small number of samples at sucb separation intervals. This approach results in an interbed range of $619 \mathrm{~m}$ for the Quadra Sand data.

A core-scale stochastic process can now be defined on the basis of the two interbed semivariograms. It is not compltationally feasible, however, to generate realizations of the size necessary for this work from core-scale data. Therefore the core-scale stochastic process is regularized over a rectangular block ( $10 \mathrm{~m}$ by $10 \mathrm{~m}$ by $30 \mathrm{~m}(x y z)$ ) in order to reduce the computational demands of the realizatio generation procedure. This block size is chosen from both computational and geologic considerations and is not comsidered to introduce a significant amount of bias into the results since the horizontal range of the core-scale semivariogram is quite large. The horizontal component of the semivariogram model fit to the regularized core-scale model is the following:

$$
\gamma(r)=0.0454\left(1-e^{-(r / 380 .)^{2}}\right)
$$

The horizontal component of the regularized semivariogram is all that is required for this study, since the focus of this work is on the ramifications of variations in transmissivity, 2 vertically integrated property. Note that the theoretical semivariogram model used in (B4) is of a double-exponenial (Gaussian) form in order to better represent the increased continuity near the origin [Journel and Huijbregts, 1978]. For a double-exponential semivariogram model, the nornut. izing parameter is equal to the range over the square root $d$ three. Also note that, as expected, the regularization proxdure has greatly reduced the variance of the semivariogra model.

A stochastic process based on the Quadra Sands data now be defined. The mean and variance, determined from the natural logarithm of the regularized hydraulic conducts. 
ny (centimeters per second), are -3.32 and 0.0454 , respecively. The semivariogram describing correlation in the borizontal plane is given by (B4). A semivariogram that is tropic in the horizontal plane is employed since there are msufficient data to characterize directional dependence in we horizontal plane. Vector notation is therefore not used in (B). The stochastic process is Gaussian (characterized by a pintly normal distribution of random variables) and secondonter stationary. This stochastic process, which serves as the base for the Monte Carlo simulations of this work, listed as case 1 in Table 1 . The other stochastic processes of Table 1 are modifications of this base case. The modifiations consist of decreasing the range of the semivariogram by 2 factor of 4 and increasing the variance up to the maximum value reported for sandy units by Freeze [1975]. Further details concerning these modifications can be found the work by Butler [1986]. Finally, it must be reemphaszed that the stochastic processes used to characterize the interbed variability of this work are assumed to represent the wionary intrinsic variability of the unit. No trends in either the mean or covariance of the random variable components of the stochastic processes are employed. This interbed vriability can be considered to represent the residual variwility after major trends have been removed. Hantush [1\%2] and Butler and Liu [1991] describe the impact of treads in transmissivity on pumping-induced drawdown.

\section{APPENDIX C}

The mathematical model employed for the pumping test simulations discussed in the text is a finite element model [Gupta et al., 1984], discrete in both space and time. The spatial and temporal discretization schemes used in this model introduce an error into the calculation of the pumpinginduced drawdown. The discretization error propagates into the transmissivities estimated from the simulated drawdown. This appendix briefly outlines the approach used in this sady to correct the estimated transmissivities for the discretization error.

The approach employed for transmissivity correction is wite straightforward. A pumping test simulation is run for the uniform aquifer case using the same spatial and temporal iscretization schemes employed in the nonuniform aquifer simulations. The simulated drawdown are analyzed using the NLS or C-J procedure, and the calculated transmissivity $\left(T_{\text {al }}\right)$ is compared to the known transmissivity of the model $\left(T_{\text {known }}\right)$. A plot of the normalized difference $\left(T_{\text {cal }}-T_{\text {known }}\right) /$ $I_{\text {knowa }}$ versus dimensionless time $\left(4 T_{\text {known }} t_{\text {initial }} / r^{2} S\right)$ is prepared, where $t_{\text {initial }}$ is the beginning time of the drawdown malysis. A separate plot is prepared for each radial location and each duration (in terms of log cycles) and method of drawdown analysis.

The basic procedure used for the correction is to estimate the oormalized difference from the plots and then apply equation (C1):

$$
T_{\text {corrected }}=\frac{T_{\text {cal }}}{1+\text { normalized difference }}
$$

Note that normalized differences can be estimated to within 0.01 for all the results reported on here. If the normalized derence cannot be estimated to within 0.01 , transmissiviare not calculated for that radial location and duration of analysis. For cases when the initial dimensionless time and the duration of the analysis are small, it may be difficult to estimate the normalized difference to within 0.01 . Thus, for example, the analysis of temporal dependence is confined to drawdown at the pumping well where the normalized differences can be estimated to within 0.0025 . For analyses of small durations using drawdown at large radial distances from the pumping well, the normalized difference cannot be estimated to within 0.1 , an unacceptably large error for this work. Note that experimental simulations indicate that the majority of the discretization error arises because of the spatial discretization scheme. A considerably finer spatial discretization scheme would be required in order to significantly reduce the uncertainty in the normalized differences for analyses of small durations beginning at small dimensionless times. The increased computational burden produced by a much finer discretization scheme was not feasible given the computer resources available to the author at the time of this study.

Finally, it must be noted that the transmissivity correction scheme used here is based on discretization error relationships developed for uniform aquifers. The focus of this work, however, is on simulations performed in nonuniform aquifers. Therefore it is necessary to assess whether the character of the discretization error for simulations in uniform units is essentially the same as that for simulations in nonuniform units. This question is addressed here by comparing the model output to an analytical solution for flow to a pumping well in a nonuniform aquifer. As described by Butler [1986], the analytical solution presented by Barker and Herbert [1982] is employed to assess the performance of the numerical model in the presence of a single radial discontinuity in transmissivity. In this case a transmissivity contrast of 2 orders of magnitude is employed, and no grid refinement is used in the vicinity of the discontinuity (radial distance $7.84 \mathrm{~m}$ ). The discretization error does not appear to be affected by the radial discontinuity. Thus it is assumed that the correction plots developed for uniform units are essentially equally applicable for nonuniform units. Note that the transmissivity contrast between neighboring elements never exceeds 2 orders of magnitude in this work.

\section{REFERENCES}

Barker, J. A., and R. Herbert, Pumping tests in patchy aquifers, Ground Water, 20(2), 150-155, 1982.

Butler, J. J., Jr., Pumping tests in nonuniform aquifers: A deterministic and stochastic analysis, Ph.D. dissertation, 220 pp., Stanford Univ., Stanford, Calif., 1986.

Butler, J. J., Jr., Pumping tests in nonuniform aquifers-The radially symmetric case, J. Hvdrol., 10l(1/4), 15-30. 1988.

Butler, J. J., Jr., The role of pumping tests in site characterization: Some theoretical considerations, Ground Water, 28(3), 394-402. 1990.

Butler, J. J., Jr., and W. Z. Liu, Analytical solutions for flow to a pumping well in nonuniform aquifers, Kans. Geol. Surv. Open File Rep. 89-32, 12 pp., 1989.

Butler, J. J., Jr., and W. Z. Liu, Pumping tests in nonuniform aquifers-The linear strip case, J. Hydrol., in press, 1991.

Cooper, H. H., Jr., and C. E. Jacob, A generalized graphical method for evaluating formation constants and summarizing wellfield history, Eos Trans. $A G U, 27(4), 526-534,1946$.

de Marsily. G., Spatial variability of properties in porous media: A stochastic approach, in Fundamentals of Transport Phenomenu in Porous Media, NATO Adv. Sti. Inst. Ser. E, vol. 82, edited by J. Bear and M. Y. Corapcioglu, pp. 719-769, Martinus Nijhoff, Dordrecht, Netherlands, 1984. 
Freeze, R. A., A stochastic-conceptual analysis of one-dimensional groundwater flow in nonuniform homogeneous media, Water Resour. Res., 11(5), 725-741, 1975.

Gupta. S. K.. C. R. Cole, F. W. Bond, and A. M. Monti, Finiteelement three-dimensional groundwater (FE3DGW) flow model: Formulation, computer source listing, and user's manual, $B M I I$ ONWI-548, 336 pp., Battelle Mem. Inst., Columbus, Ohio, 1984.

Hammersley, J. M., and D. C. Handscomb, Monte Carlo Methods, 178 pp., Methuen, London, 1964.

Hantush, M. S.. Flow of groundwater in sands of nonuniform thickness, 3, Flow to wells, J. Geophys. Res., 67(4), 1527-1534, 1962.

Huntsberger, D. V., and P. Billingsley, Elements of Statistical Inference, 349 pp., Allyn and Bacon, Boston, 1973.

Javandel, I., and P. A. Witherspoon, A method of analyzing transient fluid flow in multilayered aquifers, Water Resour. Res., $5(4), 856-869,1969$.

Joumel, A. G., and Ch. J. Huijbregts, Mining Geostatistics, 600 pp., Academic, San Diego, Calif., 1978.

Mantoglou, A., and J. L. Wilson, Simulation of random fields with the turning band method, Rep. 264, 199 pp., R. M. Parson Lab., Mass. Inst. of Technol., Cambridge, 1981.

Matheron, G., The intrinsic random functions and their applications, Adv. Appl. Probab., 5, 439-468, 1973.

McElwee, C. D., and M. A. Yukler, Sensitivity of groundwater models with respect to variations in transmissivity and storage, Water Resour. Res., 14(3), 451-459, 1978.
Miall, A. D., Principles of Sedimentary Basin Analysis. $490 \mathrm{P}$ Springer-Verlag, New York, 1984

Papadopulos, I. S., and H. H. Cooper, Jr., Drawdown in a well of large diameter, Water Resour. Res., 3(1), 241-244, 1967.

Rubinstein, R. Y., Simulation and the Monte Carlo Method, $n$ pp., John Wiley, New York, 1981.

Smith, J. L., A stochastic analysis of steady-state groundwater toon in a bounded domain, Ph.D. dissertation, 325 pp., Univ. of Brited Columbia, Vancouver, 1978.

Streltsova, T. D., Well Testing in Heterogeneous Formations, 413 pp., John Wiley, New York, 1988.

Theis, C. V., The relation between the lowering of the piezometn surface and the rate and duration of discharge of a well ground-water storage, Eos Trans. AGU, 16, 519-524, 1935.

Toth, J., Groundwater in sedimentary (clastic) rocks, Proc. Sym Ground Water Hydrol. 1967, 91-102, 1967.

Vandenberg, A., Pump testing in heterogeneous aquifers, $J$. th. drol., 34, 45-62, 1977.

Warren, J. E., and H. S. Price, Flow in heterogeneous porow media, Soc. Pet. Eng. J., I(3), 153-169, 1961.

J. J. Butler, Jr., Kansas Geological Survey, University of Kansas 1930 Constant Avenue, Lawrence, KS 66047.

(Received April 4, 1990;

revised May 9, 1991;

accepted May 14, 1991.) 